\title{
RATIONAL OFFSET SURFACES AND THEIR MODELING APPLICATIONS
}

\author{
RIMVYDAS KRASAUSKAS * AND MARTIN PETERNELL ${ }^{\dagger}$
}

\begin{abstract}
This survey discusses rational surfaces with rational offset surfaces in Euclidean 3-space. These surfaces can be characterized by possessing a field of rational unit normal vectors, and are called Pythagorean normal surfaces. The procedure of offsetting curves and surfaces is present in most modern 3d-modeling tools. Since piecewise polynomial and rational surfaces are the standard representation of parameterized surfaces in CAD systems, the rationality of offset surfaces plays an important role in geometric modeling. Simple examples show that considering surfaces as envelopes of their tangent planes is most fruitful in this context. The concept of Laguerre geometry combined with universal rational parametrizations helps to treat several different results in a uniform way. The rationality of the offsets of rational pipe surfaces, ruled surfaces and quadrics are a specialization of a result about the envelopes of one-parameter families of cones of revolution. Moreover a couple of new results are proved: the rationality of the envelope of a quadratic two-parameter family of spheres and the characterization of classes of Pythagorean normal surfaces of low parametrization degree.
\end{abstract}

Key words. rational surfaces, rational offsets, Pythagorean normal surfaces, LN surfaces, canal surfaces, Laguerre geometry, universal rational parametrization

AMS(MOS) subject classifications.

1. Introduction and the history of rational offset surfaces. When modeling real world objects one not only uses surfaces but has to take into account the material thickness. Thus offsetting curves and surfaces is a frequently used tool and it is present in most of the 3d-geometrymodeling software nowadays. These systems typically represent parameterized curves and surfaces as B-splines or piecewise rational (NURBS) curves and surfaces. This motivated several researchers $[10,7,8,9,22,20,21,27,28,34,35]$, just to name a few of them, to study rational curves and surfaces with rational offsets.

Given a parametric rational surface $\mathbf{f}(u, v)$ with unit normal vector field $\mathbf{n}(u, v)$, the offset surfaces at distance $d$ can be represented parametrically by

$$
\mathbf{f}_{d}(u, v)=\mathbf{f}(u, v)+d \mathbf{n}(u, v) .
$$

Because of the normalization of the normal vector $\mathbf{n}$, the offset surfaces of rational surfaces $\mathbf{f}(u, v)$ are typically non-rational. This also holds for curves. For instance the offsets of an ellipse are non-rational algebraic curves of degree eight. But even if the rational surface $\mathbf{f}(u, v)$ possesses rational offsets, the representation (1.1) is typically non-rational. This can already be realized for a parabola $\mathbf{c}(t)=\left(t, t^{2}\right)$, whose offsets are rational curves of degree six, but the parametrization $\mathbf{c}_{d}(t)=\mathbf{c}(t)+d \mathbf{n}(t)$ with $\mathbf{n}(t)=1 / \sqrt{1+4 t^{2}}(-2 t, 1)$ is non-rational. An appropriate reparametrization of the parabola is required to represent the offsets by rational parametrizations. Thus it is necessary to study this subject in more detail to be able to decide whether the offset surfaces of a given rational surface are rational and how to derive and construct rational parametrizations.

The rationality of a surface is determined by vanishing genus and second plurigenus. But the computation of these invariants is quite complex for surfaces given by parametric representations, such that determining the rationality of offset surfaces is difficult. Additionally we note that here we will denote a real surface as rational if and only if it admits a real rational parametrization. There exist real surfaces possessing rational (improper) parametrizations but their genus does not vanish, for instance offsets of ellipsoids.

The analogous questions for curves have been studied for a long time within the computeraided-geometric-design community. Farouki [10,7] introduced the notion of PH curves, see also the survey [8] and the recent book [9]. This term denotes polynomial curves $\mathbf{p}(t)$ with the property that the norm of the tangent vector $\dot{\mathbf{p}}(t)$ is polynomial. This implies that the arc length of $\mathbf{p}(t)$ is a polynomial. Setting $\dot{\mathbf{p}}=w(t)\left(u(t)^{2}-v(t)^{2}, 2 u(t) v(t)\right)$ with arbitrary polynomials

\footnotetext{
*Vilnius University, Lithuania, rimvydas.krasauskas@mif.vu.lt

†Vienna University of Technology, Austria, peternell@geometrie.tuwien.ac.at, Grant Austrian Science Fund FWF under project S92
} 
$u(t), v(t)$ and $w(t)$, the norm $\|\dot{\mathbf{p}}\|$ as well as the norm of the normal vector equals the polynomial $w(t)\left(u(t)^{2}+v(t)^{2}\right)$. Consequently the unit normal vector is rational. The concept of $\mathrm{PH}$ curves is also generalized to space curves, see e.g. [8].

Rational surfaces with rational offsets are more involved and the techniques used for curves do not apply to surfaces directly. An explicit representation of all rational surfaces with rational offsets has been given in [38]. Nevertheless it is not obvious how to decide the rationality of the offsets for particular classes of surfaces. It has been proved that rational pipe surfaces [28], rational ruled surfaces [42] and all regular quadrics [30] possess rational offsets. These statements can also be found in [35] as specializations of a more general result concerning envelopes of rational one-parameter families of cones of revolution.

Since any cone of revolution is the envelope of a one-parameter family of spheres as well as planes, the envelope is also generated by a two-parameter family of spheres. Using the affine space $\mathbb{R}^{4}$ as model of the four-dimensional manifold of spheres in Euclidean $\mathbb{R}^{3}$, the mentioned result reads: A rational ruled surface in the model space $\mathbb{R}^{4}$ represents a two-parameter family of spheres whose envelope surface as well as its offset surfaces possess rational parametrizations and these parametrizations can be constructed explicitly. This result is a general statement about a class of surfaces in $\mathbb{R}^{4}$ and their corresponding envelopes in $\mathbb{R}^{3}$.

The article describes the current status of research in the field of rational offset surfaces and it points to some new results and open questions. It will provide a short introduction to some theoretical tools which are necessary for their treatment. Section 2 provides a first and elementary introduction to rational offset surfaces which are constructed using the Blaschke image of the space of planes. Section 3 deals with the special family of rational surfaces possessing a 'linear normal vector field'. In Section 4 we provide a theoretical investigation of the subject introducing to Euclidean Laguerre geometry, the geometry of oriented spheres and planes in $\mathbb{R}^{3}$ and its models. Section 5 gives rational parametrizations in full generality and Section 6 deals with several special families of rational offset surfaces. Section 7 is devoted to modeling applications and finally we conclude this article and discuss some open problems.

2. Different approaches to rational offset surfaces. We start with defining rational surfaces with rational offsets, discuss these surfaces as envelopes of spheres and planes and derive concepts to obtain an elegant approach to deal with these surfaces.

Definition 2.1. A surface $F$ in $\mathbb{R}^{3}$ is a Pythagorean normal surface or PN surface if it possesses a rational parametrization $\mathbf{f}(u, v)$ and a rational unit normal vector field $\mathbf{n}(u, v)$ corresponding to $\mathbf{f}(u, v)$. The offset surface $F_{d}$ of $F$ at oriented distance $d$ admits a rational parametrization $\mathbf{f}_{d}(u, v)=\mathbf{f}(u, v)+d \mathbf{n}(u, v)$.

The rationality of a surface does not depend on a particular parametrization. Since we take a constructive viewpoint we like to construct parametrizations $\mathbf{f}(u, v)$ which directly lead to rational parametrizations $\mathbf{f}_{d}(u, v)$ of the offsets. The correspondence noted in Definition 2.1 means that $\mathbf{n}(u, v)$ is computed via normalizing the cross product $\mathbf{f}_{u} \times \mathbf{f}_{v}$,

$$
\mathbf{n}(u, v)=\frac{1}{\left\|\mathbf{f}_{u}(u, v) \times \mathbf{f}_{v}(u, v)\right\|} \mathbf{f}_{u}(u, v) \times \mathbf{f}_{v}(u, v),
$$

with $\mathbf{f}_{u}$ and $\mathbf{f}_{v}$ as partial derivatives of $\mathbf{f}$ with respect to $u$ and $v$, respectively. If the norm $\left\|\mathbf{f}_{u}(u, v) \times \mathbf{f}_{v}(u, v)\right\|$ is a rational function, the parametrization (1.1) is a rational representation of the offsets $F_{d}$ of $F$ and is called $P N$-parametrization. But typically this norm involves square roots even for rational offset surfaces and appropriate reparametrizations have to be performed.

2.1. Offsets as envelopes of spheres and planes. Assuming $\mathbf{f}(u, v)$ is a PN-parametrization of a rational offset surface $F$, the tangent planes $E(u, v)$ of $F$ admit the rational representation

$$
E(u, v): n_{0}(u, v)+\mathbf{n}(u, v) \cdot \mathbf{x}=0, \text { with }\|\mathbf{n}(u, v)\|^{2}=1 .
$$

The rational support function $n_{0}=-\mathbf{n} \cdot \mathbf{f}$ expresses the oriented distance of the origin from $E$. Eqn. (2.2) interprets the surface $F$ as the envelope of the two-parameter family of tangent planes 
$E(u, v)$. Likewise let the offset surface $F_{d}$ of $F$ be considered as the envelope of its tangent planes. Translating the planes $E$ by the constant oriented distance $d$ in direction of the normal vector $\mathbf{n}$ results in the tangent planes $E_{d}$ of the offset surface $F_{d}$, with

$$
E_{d}(u, v): n_{0}(u, v)-d+\mathbf{n}(u, v) \cdot \mathbf{x}=0 .
$$

Given a parameterized surface $F$ with representation $\mathbf{f}(u, v)$, the offset surface $F_{d}$ at distance $d$ can be considered as the envelope of a two-parameter family of spheres

$$
S:(\mathbf{x}-\mathbf{f}(u, v)) \cdot(\mathbf{x}-\mathbf{f}(u, v))-d^{2}=0,
$$

of radius $d$ which are centered at the surface $F$. According to the envelope condition an implicit equation of the offset surface $F_{d}$ is obtained by eliminating the surface parameters $u$ and $v$ from the system of equations

$$
S:(\mathbf{x}-\mathbf{f}) \cdot(\mathbf{x}-\mathbf{f})-d^{2}=0, \quad \frac{\partial S}{\partial u}:(\mathbf{x}-\mathbf{f}) \cdot \mathbf{f}_{u}=0, \quad \frac{\partial S}{\partial v}:(\mathbf{x}-\mathbf{f}) \cdot \mathbf{f}_{v}=0 .
$$

Note that these two approaches are not equivalent. The first interpretation (2.3) yields one-sided offsets whereas the second one (2.5) results typically in two sheets of the offset surface at both sides of $F$. If we consider rational surfaces, both approaches might yield the same result if the original surface $F$ is traced twice and thus both orientations of the normal vector field $\mathbf{n}(u, v)$ appear.

Now we focus on the interpretation of offset surfaces as the envelopes of their tangent planes (2.3). For this reason we introduce to the manifold of oriented planes of $\mathbb{R}^{3}$. Later we will see the close connection between oriented planes and spheres in Section 4.

2.2. The space of oriented planes and the Blaschke model. We consider the family of oriented planes $E: e_{0}+\mathbf{e} \cdot \mathbf{x}=0$, where e denotes the unit normal vector of $E$, similar to (2.2). The real numbers $e_{0}$, and $\mathbf{e}=\left(e_{1}, e_{2}, e_{3}\right)$ determine the oriented plane. We use $\left(e_{1}, e_{2}, e_{3}, e_{0}\right) \in \mathbb{R}^{4}$ with $\|\mathbf{e}\|=1$ as coordinates of oriented planes. Denoting the family of planes of $\mathbb{R}^{3}$ by $\mathcal{E}$, this defines the Blaschke mapping

$$
\beta: \mathcal{E} \rightarrow \mathbb{R}^{4}, \quad E: e_{0}+\mathbf{e} \cdot \mathbf{x}=0 \mapsto \beta(E)=\left(e_{1}, e_{2}, e_{3}, e_{0}\right),
$$

which identifies oriented planes $E \in \mathcal{E}$ of $\mathbb{R}^{3}$ with the family of points $\beta(E) \in \mathbb{R}^{4}$. According to the normalization condition $\|\mathbf{e}\|=1$ the image points $\beta(E)$ are contained in the quadratic cone

$$
\mathcal{B}: y_{1}^{2}+y_{2}^{2}+y_{3}^{2}=1 \text {, }
$$

called the Blaschke cylinder $\mathcal{B}$. Here we use $y_{1}, \ldots, y_{4}$ as Cartesian coordinates in $\mathbb{R}^{4}$.

The intersections of $\mathcal{B}$ with 3 -spaces $y_{4}=$ constant are copies of the unit sphere $S^{2}: x_{1}^{2}+$ $x_{2}^{2}+x_{3}^{2}=1$ and $\mathcal{B}$ is a cylinder over $S^{2}$. Consider two parallel planes $E: e_{0}+\mathbf{n} \cdot \mathbf{x}=0$ and $F: f_{0}+\mathbf{n} \cdot \mathbf{x}=0$ with coinciding unit normal $\mathbf{n}$. Their image points $\beta(E)$ and $\beta(F)$ are contained in a generating line of $\mathcal{B}$.

2.3. The Blaschke image of a PN surface. The introduction of the Blaschke mapping provides a theoretical background for the study of PN surfaces and applies also to find rational parametrizations of these surfaces.

Let $F$ be a PN surface with representation $\mathbf{f}(u, v)$ and tangent planes $E(u, v): e_{0}(u, v)+$ $\mathbf{e}(u, v) \cdot \mathbf{x}=0$. The unit normal vector field $\mathbf{e}(u, v)$ is a rational parametrization of the unit sphere $S^{2}$. The Blaschke image $\beta(F)=\beta(E(u, v))$ is a rational surface in $\mathcal{B}$ with rational parametrization $\beta(E)=\left(e_{1}, e_{2}, e_{3}, e_{0}\right)(u, v)$.

THEOREM 2.1. The Blaschke image $\beta(F)$ of a $P N$ surface $F$ is a rational surface in $\mathcal{B}$. Conversely, any rational surface in $\mathcal{B}$ is the Blaschke image of a $P N$ surface.

Given any rational two-dimensional surface $S$ in the Blaschke cylinder $\mathcal{B}$, we have to consider it as image points of tangent planes. The envelope of this two-parameter family of planes corresponding to the surface $S \subset \mathcal{B}$ is a PN surface in $\mathbb{R}^{3}$. Let $\mathbf{S}(u, v)=\left(s_{1}, \ldots, s_{4}\right)(u, v)$ be a rational parametrization of $S$. The corresponding family of tangent planes is

$$
T(u, v): s_{4}(u, v)+\mathbf{s}(u, v) \cdot \mathbf{x}=0,
$$


where $\mathbf{s}=\left(s_{1}, s_{2}, s_{3}\right)$ denotes the unit normal vector of $T$. Computing the partial derivatives $T_{u}(u, v)$ and $T_{v}(u, v)$ of $T(u, v)$ gives a PN-parametrization $\mathbf{f}(u, v)$ of the surface $F$ as solution of the system of linear equations

$$
\begin{array}{r}
T(u, v): s_{4}(u, v)+\mathbf{s}(u, v) \cdot \mathbf{x}=0, \\
T_{u}(u, v): s_{4_{u}}(u, v)+\mathbf{s}_{u}(u, v) \cdot \mathbf{x}=0, \\
T_{v}(u, v): s_{4 v}(u, v)+\mathbf{s}_{v}(u, v) \cdot \mathbf{x}=0 .
\end{array}
$$

Until now we have restricted our interest to two-dimensional surfaces in $\mathcal{B}$. But what about curves? Consider a rational curve $C \subset \mathcal{B}$ with representation $\mathbf{C}(t)=\left(c_{1}, \ldots, c_{4}\right)(t)$. The corresponding surface $\beta^{-1}(C)=D$ is the envelope of a one-parameter family of planes and thus a developable PN surface. The generating lines $g(t)$ are obtained as intersections $T \cap T_{t}$ and are the solutions of the system of equations

$$
\begin{gathered}
T(t): c_{4}(t)+\mathbf{c}(t)^{T} \cdot \mathbf{x}=0, \\
T_{t}(t): c_{4 t}(t)+\mathbf{c}_{t}(t)^{T} \cdot \mathbf{x}=0 .
\end{gathered}
$$

By the way, the intersection $\mathbf{v}(t)=T \cap T_{t} \cap T_{t t}$ is in general the singular curve $V$ of $D$. For special developable surfaces like cones and cylinders, $V$ degenerates to a point or an ideal point, respectively. We summarize these results.

TheOREm 2.2. The Blaschke image $\beta(F)$ of a developable PN surface $F$ is a rational curve in $\mathcal{B}$. Conversely, any rational curve in $\mathcal{B}$ is the Blaschke image of a developable PN surface. The Theorems 2.1 and 2.2 can be found in a different form in [35]. There the stereographic projection of the Blaschke cylinder to a 3-space and the corresponding projections of the curves and surfaces $\beta(F)$ are investigated.

In the sequel we do not pay much attention to developable surfaces and thus we assume that the family of tangent planes of considered surfaces is two-dimensional, unless explicitly mentioned.

2.4. The Gaussian image of a PN surface. We consider a rational offset surface $F \subset \mathbb{R}^{3}$ whose tangent planes have the form $T(u, v): e_{0}(u, v)+\mathbf{e}(u, v) \cdot \mathbf{x}=0$, with $\|\mathbf{e}\|=1$. The Blaschke image $\beta(F)=\left(e_{1}, e_{2}, e_{3}, e_{0}\right)$ of $F$ consists of

- a rational parametrization $\mathbf{e}(u, v)$ of the unit sphere $S^{2}$, and

- the support function $e_{0}(u, v)$ of $F$.

The parametrization $\mathbf{e}(u, v)$ of the unit normal vector field of $F$ is the Gaussian image of $F$. One can take apart the Blaschke image $\beta(F)$ and study the Gaussian image $\mathbf{e}(u, v)$ and the support function $e_{0}(u, v)$ separately. This constructive approach to PN surfaces is based on the study of rational parametrizations of the unit sphere through the Gauss map.

2.5. Rational parametrizations of the unit sphere via stereographic projection. The easiest way to construct rational parametrizations of unit sphere $S^{2}$ is as follows. Let $a(u, v), b(u, v)$ and $c(u, v)$ be relatively prime bivariate polynomials. Then a rational parametrization $\mathbf{e}=\left(e_{1}, e_{2}, e_{3}\right)$ of $S^{2}$ is obtained by

$$
e_{1}=\frac{2 a c}{n}, e_{2}=\frac{2 b c}{n}, e_{3}=\frac{a^{2}+b^{2}-c^{2}}{n}, \text { with } n=a^{2}+b^{2}+c^{2} .
$$

This parametrization is a composition of a rational parametrization $\mathbf{x}=\left(\frac{a}{c}, \frac{b}{c}\right)$ of $\mathbb{R}^{2}$ and the stereographic projection $\sigma: \mathbb{R}^{2} \rightarrow S^{2}$ with center $(0,0,1)$

$$
\sigma(\mathbf{x})=\left(\frac{2 x_{1}}{x_{1}^{2}+x_{2}^{2}+1}, \frac{2 x_{2}}{x_{1}^{2}+x_{2}^{2}+1}, \frac{x_{1}^{2}+x_{2}^{2}-1}{x_{1}^{2}+x_{2}^{2}+1}\right) .
$$

The parametrization (2.10) is geometrically evident but has the drawback that it is dependent not only on the coordinate system but also on the choice of the center for the stereographic projection. To avoid this we will introduce universal rational parametrizations of $S^{2}$ in Section 5 . This leads to universal parametrizations of the Blaschke cylinder $\mathcal{B} \subset \mathbb{R}^{4}$ which represent the most general approach to PN surfaces. 
2.6. Rational parametrizations of $\mathbf{P N}$ surfaces in the simple form. Starting from the parametrization (2.10) of the unit sphere one can derive rational parametrizations of $\mathrm{PN}$-surfaces as already developed in [38]. Prescribing a rational function $h(u, v)=f(u, v) / g(u, v)$, the tangent planes of a rational offset surface take the form

$$
T(u, v): h+\frac{2 a c}{n} x_{1}+\frac{2 b c}{n} x_{2}+\frac{a^{2}+b^{2}-c^{2}}{n} x_{3}=0 .
$$

Multiplying by the denominator $n$ gives a polynomial representation of the tangent planes in the form

$$
T(u, v): f n+2 a c g x_{1}+2 b c g x_{2}+g\left(a^{2}+b^{2}-c^{2}\right) x_{3}=0,
$$

where $f$ and $g$ are polynomials without a common factor. This approach is best illustrated by an example.

EXAmple 1. Assume that $a=u, b=v$ and $c=1$. This leads to the standard form of $a$ rational parametrization of $S^{2}$ by

$$
\left(\frac{2 u}{u^{2}+v^{2}+1}, \frac{2 v}{u^{2}+v^{2}+1}, \frac{u^{2}+v^{2}-1}{u^{2}+v^{2}+1}\right) .
$$

Further we assume $g=1$ and choose $f$ as an arbitrary quadratic polynomial $q(u, v)$. The surfaces whose tangent planes can be parameterized by

$$
T(u, v): q(u, v)+2 u x_{1}+2 v x_{2}+\left(u^{2}+v^{2}-1\right) x_{3}=0
$$

are known as parabolic Dupin cyclides. These surfaces are of algebraic order 3 and form a family of surfaces which is closed under taking offsets (see Fig. 1). The real singularities of the surfaces and their offsets might be different.
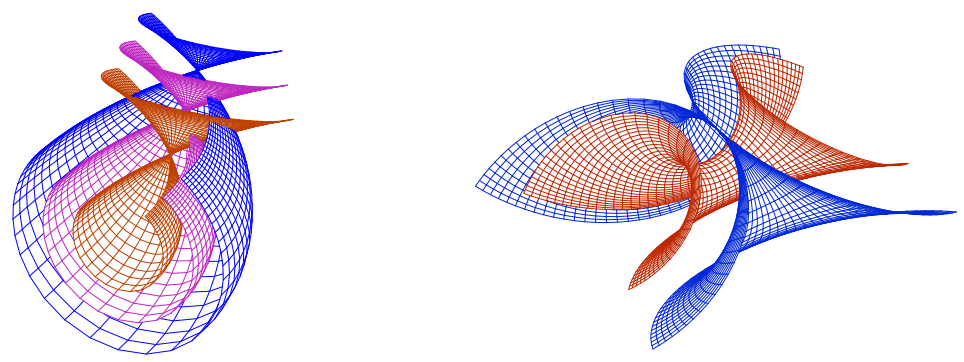

FIG. 1. Parabolic Dupin cyclides and their offsets

3. Rational Surfaces with a linear normal vector field. A special class of rational offset surfaces is formed by the offsets of rational surfaces which possess a linear normal vector field, see [13]. Moreover it has been shown in [48] that the convolutions of these surfaces with all rational surfaces are again rational.

A rational surface $F$ is called an $L N$ surface if there exists a rational parametrization $\mathbf{s}(u, v)$ such that a normal vector field $\mathbf{n}(u, v)$ of $S$ can be linearly parameterized as

$$
\mathbf{n}(u, v)=\mathbf{p} u+\mathbf{q} v+\mathbf{r}, \text { with } \mathbf{p}, \mathbf{q}, \mathbf{r} \in \mathbb{R}^{3} .
$$

Note that the normal vector field $\mathbf{n}(u, v)$ is in general not normalized and not oriented. This is quite different from the previous approach to rational offset surfaces via PN surfaces in Section 2.3. Later on we show how this class of surfaces fits to the presented concept and how the Blaschke images $\beta(F)$ of LN surfaces look like.

In the following we assume that $\operatorname{rank}(\mathbf{p}, \mathbf{q}, \mathbf{r})=3$, the coordinate vectors $\mathbf{n}(u, v)$ parameterize points of an affine plane. This implies that the unit normal vectors of $F$ parameterize a twodimensional subset of $S^{2}$. Otherwise the corresponding surface $F$ is either a cylinder or a plane. 
An appropriate choice of the coordinate system is $\mathbf{p}=(1,0,0), \mathbf{q}=(0,1,0)$, and $\mathbf{r}=(0,0,1)$, and the normal vector becomes $\mathbf{n}(u, v)=(u, v, 1)^{T}$, which we assume below. The tangent planes $T(u, v)$ of an LN surface $F$ have the quite simple representation

$$
T(u, v): h(u, v)+u x+v y+z=0,
$$

where $h(u, v)$ is a rational function. With respect to the chosen coordinate system, the tangent planes $T$ are graphs of linear functions over the $x y$-plane. The representation (3.2) allows it to treat $(u, v, h(u, v))$ as affine coordinates of $T$. Using $(U, V, W)$ as coordinate functions of planes, the dual affine equation of an LN surface $F$ is $W=h(U, V)$. This representation says that the tangent planes of LN surfaces are graphs of rational functions.

This property has the following important consequence: For any vector $\mathbf{n}=(u, v, 1)^{T}$ there exists a unique tangent plane $T(u, v)$ of $F$ having $\mathbf{n}$ as normal vector and there exists exactly one point of contact of $F$ and $T$. This unique-tangent-plane-property is the reason for the rationality of the convolution surfaces with any arbitrary rational surface, see [48]. Summarizing we obtain

Corollary 3.1. The family of tangent planes $T(u, v)$ of an $L N$ surface $F$ can be represented in plane coordinates by the graph $(u, v, h(u, v))$ of a rational function $h$. Conversely, the graph of a rational function represents the tangent planes (3.2) of an LN surface. The convolution surface $F \star G$ of an $L N$ surface $F$ and any arbitrary rational surface $G$ is a rational surface.

3.1. The Blaschke image of $\mathbf{L N}$ surfaces. Since LN surfaces $F$ are very special concerning their normal vectors and tangent planes, also their Blaschke image $\beta(F)$ is of a special kind. To obtain rational parametrizations of their Blaschke image $\beta(F)$ we have to reparameterize and normalize the unit normal vector field. Inserting the rational reparametrization

$$
u=\frac{2 s}{1-s^{2}-t^{2}}, v=\frac{2 t}{1-s^{2}-t^{2}}
$$

into the representation (3.2) and normalizing the normal vector leads to

$$
T(s, t): \frac{1-s^{2}-t^{2}}{1+s^{2}+t^{2}} h(s, t)+\frac{2 s}{1+s^{2}+t^{2}} x+\frac{2 t}{1+s^{2}+t^{2}} y+\frac{1-s^{2}-t^{2}}{1+s^{2}+t^{2}} z=0,
$$

which exhibits that LN surfaces are rational offset surfaces (PN surfaces). The reparametrization (3.3) induces an orientation to the plane $T(s, t)$ as well as to the surface $F$. The substitution (3.3) is not one-to-one, but there exist parameters

$$
s^{\prime}=\frac{-s}{s^{2}+t^{2}}, \text { and } t^{\prime}=\frac{-t}{s^{2}+t^{2}},
$$

for which $u(s, t)=u\left(s^{\prime}, t^{\prime}\right)$ and $v(s, t)=v\left(s^{\prime}, t^{\prime}\right)$ holds. The normal vector $\mathbf{n}(u, v)=(u, v, 1)$ corresponds to and is parallel to the two oriented unit normal vectors

$$
\mathbf{n}(s, t)=-\mathbf{n}\left(s^{\prime}, t^{\prime}\right)=\left(\frac{2 s}{1+s^{2}+t^{2}}, \frac{2 t}{1+s^{2}+t^{2}}, \frac{1-s^{2}-t^{2}}{1+s^{2}+t^{2}}\right)=\left(n_{1}, n_{2}, n_{3}\right) .
$$

This further implies that the function $h$ satisfies $h(s, t)=h\left(s^{\prime}, t^{\prime}\right)$. Putting things together we see that $T(s, t)$ and

$$
T\left(s^{\prime}, t^{\prime}\right):-\frac{1-s^{2}-t^{2}}{1+s^{2}+t^{2}} h\left(s^{\prime}, t^{\prime}\right)-\left(n_{1} x+n_{2} y+n_{3} z\right)=0
$$

describe the same carrier plane but have different orientations according to oppositely pointing unit normals $\mathbf{n}(s, t)=-\mathbf{n}\left(s^{\prime}, t^{\prime}\right)$. Finally we realize that the Blaschke image $\beta(F)=\left(n_{1}, n_{2}, n_{3}, n_{0}\right)$ of an LN surface has the special property that $n_{0}$ is a rational function over the unit sphere $S^{2}$ satisfying $n_{0}(\mathbf{x})=-n_{0}(-\mathbf{x})$, if $\mathbf{x}$ and $-\mathbf{x}$ are antipodal points in $S^{2}$.

COROLLARY 3.2. Let $F$ be an LN surface whose tangent planes $T$ are given by (3.2). There exists a reparametrization (3.3) which shows the rational offset property of LN surfaces. The Blaschke image $\beta(F)=\left(\mathbf{n}, n_{0}\right)$ is a function over $S^{2}$ which satisfies $n_{0}(\mathbf{x})=-n_{0}(-\mathbf{x})$ for $\mathbf{x} \in S^{2}$. 
4. Laguerre geometry approach. Thinking about surface offsets as wave fronts in different time moments naturally leads to the notion of 4-dimensional Minkowski space. This section explains duality between the Gaussian sphere and the Blaschke cylinder in terms of projective Minkowski space and its dual. Relations with the three main models of the classical Laguerre geometry are established.

4.1. Gaussian sphere in projective Minkowski space. The classical 4-dimensional Minkowski space $\mathcal{M}$ is an affine space $\mathbb{R}^{4}$ with a Minkowski scalar product defined for every pair of vectors $\mathbf{v}$ and $\mathbf{w}$ by

$$
\langle\mathbf{v}, \mathbf{w}\rangle=v_{1} w_{1}+v_{2} w_{2}+v_{3} w_{3}-v_{4} w_{4}
$$

A vector $\mathbf{v}$ (or a line with a direction $\mathbf{v}$ ) is called isotropic (light-like) if $\langle\mathbf{v}, \mathbf{v}\rangle=0$. The Euclidean space $\mathbb{R}^{3}$ will be embedded as hyperplane $x_{4}=0$ in $\mathcal{M}$. Restricting the Minkowski scalar product (4.1) to $\mathbb{R}^{3}$ gives the Euclidean scalar product.

The projective Minkowski space $\mathcal{M P}$ is a projective closure of $\mathcal{M}$ with the infinite hyperplane $\omega: x_{0}=0$ containing the absolute quadric $\Omega: x_{1}^{2}+x_{2}^{2}+x_{3}^{2}-x_{4}^{2}=0$. Points and vectors of the affine Minkowski space $\mathcal{M}$ will be treated differently. Points $\left(x_{1}, \ldots, x_{4}\right) \in \mathcal{M}$ will be identified with points $\left[1, x_{1}, \ldots, x_{4}\right]$ in the affine part of $\mathcal{M} P$, and vectors $\left(x_{1}, \ldots, x_{4}\right) \in \mathcal{M}$ will be used to represent points $\left[0, x_{1}, \ldots, x_{4}\right]$ in $\omega$. Then the equation of $\Omega \subset \omega$ has a compact form $\langle\mathbf{v}, \mathbf{v}\rangle=0$, for vectors $\mathbf{v}$ in $\mathcal{M}$. Futhermore, it will be convenient to identify the absolute quadric $\Omega$ with the Gaussian sphere $S^{2}$ in $\mathbb{R}^{3}$ using the following bijective correspondence:

$$
S^{2} \rightarrow \Omega, \quad \mathbf{n} \mapsto \mathbf{n}^{+}=(\mathbf{n}, 1)
$$

An oriented surface $F^{\circ}$ in $\mathbb{R}^{3}$ is a surface $F$ and a field of unit normals $\mathbf{n}: \mathbf{x} \mapsto \mathbf{n}(\mathbf{x})$, i.e. the classical Gaussian map $\mathbf{n}: F \rightarrow S^{2}$. By the identification (4.2) this is a map $\mathbf{n}^{+}: F \rightarrow \Omega$. Define an isotropic hypersurface $\Gamma\left(F^{\circ}\right) \subset \mathcal{M} P$ as a union of isotropic lines connecting all points $\mathbf{x} \in F$ with $\mathbf{n}^{+}(\mathbf{x}) \in \Omega$. The affine part of any such line can be parametrized by $\mathbf{x}+t \mathbf{n}^{+}(\mathbf{x}), t \in \mathbb{R}$. Hence the orthogonal projection of any hyperplane section $\Gamma\left(F^{\circ}\right) \cap\left\{x_{4}=d\right\}$ to $\mathbb{R}^{3}$ is exactly the offset $F_{d}^{\circ}$ of $F^{\circ}$ at the signed distance $d$.

The isotropic hypersurface $\Gamma\left(S^{\circ}\right)$ of an oriented sphere $S^{\circ}:(\mathbf{x}-\mathbf{m})^{2}=r^{2}$ in $\mathbb{R}^{3}$ is the union of all isotropic lines intersecting at the point $\mathbf{s}=(\mathbf{m}, \pm r) \in \mathcal{M}$, where $\mathbf{m}$ is the center and $|r|$ is the radius of $S$. It is easy to check that the outward pointing normals correspond to negative radius (in other sources of Laguerre geometry this choice might be opposite). Therefore, $\Gamma\left(S^{\circ}\right)$ coincides with the isotropic cone $\Gamma(\mathbf{s})$ with vertex $\mathbf{s}$ defined by the equation

$$
\Gamma(\mathbf{s}):\langle\mathbf{x}-\mathbf{s}, \mathbf{x}-\mathbf{s}\rangle=0 .
$$

Laguerre transformations of $\mathcal{M P}$ are projective transformations that preserve $\Omega$. Lines in $\mathcal{M P}$ are called space-like, isotropic, or time-like depending whether their ideal points are outside of $\Omega$, at $\Omega$ or is inside the quadric $\Omega$. Similarly planes and hyperplanes in $\mathcal{M} P$ are classified as space-like, isotropic, or time-like if they do not intersect $\Omega$, are tangent to $\Omega$ or intersect $\Omega$ in more than one point, respectively.

For an oriented plane $E^{\circ}: e_{0}+\mathbf{e}^{T} \cdot \mathbf{x}=0$ in $\mathbb{R}^{3}$ with normal vector $\mathbf{e},\|\mathbf{e}\|=1$, its isotropic hypersurface $E^{+}=\Gamma\left(E^{\circ}\right)$ is the hyperplane

$$
E^{+}: e_{0}+\left\langle\mathbf{e}^{+}, \mathbf{x}\right\rangle=0, \mathbf{x} \in \mathcal{M}
$$

Let $-E^{\circ}:-e_{0}-\mathbf{e}^{T} \cdot \mathbf{x}=0$ be the same plane $E$ with the opposite orientation. Then $E^{-}=\Gamma\left(-E^{\circ}\right)$ is different from $E^{+}$. Both $E^{+}$and $E^{-}$are the unique isotropic hyperplanes that contain $E$ and are tangent to $\Omega$.

4.2. Dual projective Minkowski space and the Blaschke cylinder. Let $\mathcal{M} P^{*}$ be the space dual to the projective Minkowski space $\mathcal{M} P$. Points in $\mathcal{M} P^{*}$ are hyperplanes $H \subset \mathcal{M} P$. The 
set of oriented planes in $\mathbb{R}^{3}$ is in 1-1 correspondence $E^{\circ} \mapsto E^{+}(4.4)$ with the set of hyperplanes in $\mathcal{M P}$ that are tangent to $\Omega$. The latter set by duality

$$
\Gamma\left(E^{\circ}\right): e_{0}+\left\langle\mathbf{e}^{+}, \mathbf{x}\right\rangle=0 \mapsto\left[1, \mathbf{e}, e_{0}\right]=\beta\left(E^{\circ}\right)
$$

defines the dual quadric $\Omega^{*} \subset \mathcal{M} P^{*}$ with the equation $y_{0}^{2}=y_{1}^{2}+y_{2}^{3}+y_{3}^{2}$. The affine part $y_{0} \neq 0$ of $\Omega^{*}$ is exactly the Blaschke cylinder defined in Section 2.2 by the equation (2.7). Note that $\Omega^{*}$ has just one additional real point $[0,0,0,1]$ at infinity. Therefore, it is natural to call the dual Gaussian sphere $\Omega^{*}$ the Blaschke cylinder and denote it by the same letter $\mathcal{B}$.

An oriented surface $F^{\circ}$ considered as family of oriented tangent planes, defines a surface $\beta\left(F^{\circ}\right) \subset \mathcal{B}$, which is called the Blaschke image of $F^{\circ}$. Going back to the point representation, one can check that the dual of the Blaschke image $\beta\left(F^{\circ}\right)^{*}$ is an isotropic hypersurface $\Gamma\left(F^{\circ}\right)$. This means that $\Gamma\left(F^{\circ}\right)$ can be calculated as envelope of all isotropic hyperplanes $H, H \in \beta\left(F^{\circ}\right)^{*}$.

For any surface $\Phi$ or curve in $\mathcal{M}$, define the isotropic hypersurface $\Gamma(\Phi)=\left(\Phi^{*} \cap \mathcal{B}\right)^{*}$. This is the envelope of all isotropic hyperplanes tangent to both $\Phi$ and $\Omega . \Gamma(\Phi)$ can be calculated as an envelope of all isotropic cones $\Gamma(\mathbf{x}), \mathbf{x} \in \Phi$, as well. The cyclographic image of $\Phi \subset \mathcal{M}$ in $\mathbb{R}^{3}$ is defined as intersection $\gamma(\Phi)=\Gamma(\Phi) \cap \mathbb{R}^{3}$.

REMARK 4.1. In general $\gamma(\Phi)$ is an oriented surface of two sheets. Indeed, any of its tangent planes inherits orientation from the unique isotropic tangent hyperplane of $\Gamma(\Phi)$ at the same point. There are exactly two tangent hyperplanes at any point of $\Phi$ (it is a double surface of $\Gamma(\Phi)$ ). For example, if $F_{1}^{\circ}$ and $F_{2}^{\circ}$ are two oriented surfaces in $\mathbb{R}^{3}$ then $\Phi=\Gamma\left(F_{1}^{\circ}\right) \cap \Gamma\left(F_{2}^{\circ}\right)$ is a surface in $\mathcal{M}$, and $\gamma(\Phi)=F_{1}^{\circ} \cup F_{2}^{\circ}$. The case of two cylinders will be considered in Example 6. In a recent paper [17] sufficient conditions are derived when a rational parametrization of $\Phi$ generates $P N$ parametrizations on $F_{1}^{\circ}$ and $F_{2}^{\circ}$.

4.3. Three models of Laguerre geometry. The classical Laguerre geometry has three main models that are described in the following table.

\begin{tabular}{l|ccc}
\hline & Euclidean model & Cyclographic model & Blaschke model \\
\hline \hline Ambient space & Euclidean space $\mathbb{R}^{3}$ & Minkowski space $\mathcal{M}$ & Blaschke cylinder $\mathcal{B}$ \\
\hline Basic elements & oriented planes $E^{\circ}$ & isotropic hyperplanes & points \\
& oriented spheres $S^{\circ}$ & points & hyperplane sections \\
\hline Basic relations & oriented contact & incidence & incidence \\
\hline
\end{tabular}

The correspondence between the Euclidean and the cyclographic model is defined by the maps $E^{\circ} \mapsto E^{+}$and $S^{\circ} \mapsto \Gamma\left(S^{\circ}\right)=\Gamma(\mathbf{x}), \mathbf{x} \in \mathcal{M}$. The cyclographic mapping $E^{+} \mapsto \gamma\left(E^{+}\right)$and $\mathbf{x} \mapsto \gamma(\mathbf{x})$ establishes the inverse correspondence. Thereby $\gamma\left(E^{+}\right)=E^{\circ}$ denotes the oriented plane $E^{\circ} \in \mathbb{R}^{3}$ corresponding to $E^{+} \in \mathcal{M}$ and $\gamma(\mathbf{x})$ denotes the oriented sphere in $\mathbb{R}^{3}$ corresponding to the point $\mathbf{x} \in \mathcal{M}$. For the computation of the cyclographic image $\gamma(\Phi)$ of a surface $\Phi \in \mathcal{M}$ see Section 4.4 .

The Blaschke model is focusing on the dual point of view. It is related to the cyclographic model via duality. Laguerre transformations appear in the cyclographic model as special affine transformations of $\mathcal{M}$

$$
L(\mathbf{x})=\lambda A \cdot \mathbf{x}+\mathbf{a}, \text { with } A^{T} \cdot I_{c} \cdot A=I_{c}, I_{c}=\operatorname{diag}(1,1,1,-1), \lambda \in \mathbb{R}
$$

where $\mathbf{x} \mapsto A \mathbf{x}$ is a Lorentz transformation, i.e. a linear transformation preserving the Minkowski scalar product (4.1). Laguerre transformations in the Blaschke model are defined by duality.

Laguerre transformations in the Euclidean model can be defined indirectly as follows. For an oriented surface $F^{\circ} \subset \mathbb{R}^{3}$, its Laguerre transformation $L\left(F^{\circ}\right)$ is computed by the formula $L\left(F^{\circ}\right)=L\left(\Gamma\left(F^{\circ}\right)\right) \cap \mathbb{R}^{3}$. For example, let $L$ be the translation in $x_{4}$-direction by $-d: x_{4} \mapsto x_{4}-d$. Then $L\left(F^{\circ}\right)=F_{d}^{\circ}$ is the offset surface of $F^{\circ}$ at distance $d$.

4.4. Cyclographic images of parametric curves and surfaces in $\mathcal{M}$. A two-parameter family of spheres $S(u, v):(\mathbf{x}-\mathbf{m}(u, v))^{2}=r(u, v)^{2}$ with centers $\mathbf{m}(u, v)$ and radii $r(u, v)$ corresponds to a parametrized surface $\Phi: \mathbf{f}(u, v)=(\mathbf{m}, r)(u, v)$ in $\mathcal{M}$. Let $F(u, v)=\Gamma(\mathbf{f}(u, v))$ be 
the corresponding two-parameter family of isotropic cones with vertices $\mathbf{f}(u, v)$. Then $\Gamma(\Phi)$ is the envelope of this family, which can be computed as solution of

$$
\begin{array}{r}
F:\langle\mathbf{x}-\mathbf{f}, \mathbf{x}-\mathbf{f}\rangle=0, \\
F_{u}:\left\langle\mathbf{x}-\mathbf{f}, \mathbf{f}_{u}\right\rangle=0, \\
F_{v}:\left\langle\mathbf{x}-\mathbf{f}, \mathbf{f}_{v}\right\rangle=0,
\end{array}
$$

where $F_{u}$ and $F_{v}$ denote the partial derivatives of $F$ with respect to $u$ and $v$. The solution of (4.7) consists of all isotropic lines that are orthogonal to $\Phi$ in the Minkowski sense (4.1).

Comparing systems (4.7) and (2.5) we recognize that the cyclographic image $\gamma(\Phi)=\Gamma(\Phi) \cap \mathbb{R}^{3}$ of the parametrized surface $\Phi: \mathbf{f}(u, v)=(\mathbf{m}, r)(u, v)$ is the envelope of the two-parameter system of spheres $S(u, v)$ in $\mathbb{R}^{3}$. We note that only the points $\mathbf{f}$ of $\Phi$ whose tangent planes $T_{f}$ spanned by $\mathbf{f}_{u}$ and $\mathbf{f}_{v}$ are space-like or isotropic, will contribute to the real part of the cyclographic image $\gamma(\Phi)$.

A one-parameter family of spheres $S(t):(\mathbf{x}-\mathbf{m}(t))^{2}=r(t)^{2}$ corresponds to a curve $\mathbf{s}(t)=$ $(\mathbf{m}, r)(t)$ in $\mathcal{M}$. By similar calculations the isotropic hypersurface $\Gamma(\mathbf{s})$ consists of all isotropic lines that intersect the curve orthogonally. The cyclographic image $\gamma(\mathbf{s})=\Gamma(\mathbf{s}) \cap \mathbb{R}^{3}$ is the envelope of the family of spheres $S(t)$ and is called canal surface. It is real exactly if tangent vectors $\dot{\mathbf{s}}$ are space-like or isotropic

$$
\langle\dot{\mathbf{s}}(t), \dot{\mathbf{s}}(t)\rangle=\|\dot{\mathbf{m}}(t)\|^{2}-\dot{r}(t)^{2} \geq 0 .
$$

5. Universal rational parametrizations of the sphere and the Blaschke cylinder. Dietz, Hoschek and Jüttler [6] noticed that Bézier curves and surface patches on the unit sphere $S^{2}$ can be represented uniformly by introducing the generalized stereographic projection $\delta: \mathbb{R}^{4} \rightarrow S^{2}$

$$
\delta(a, b, c, d)=\left(a^{2}+b^{2}+c^{2}+d^{2}, 2 a c+2 b d, 2 b c-2 a d, a^{2}+b^{2}-c^{2}-d^{2}\right) .
$$

In complex notations $z=a+b \mathrm{i}, w=c+d \mathrm{i}$ this construction has the following form [18]

$$
P_{S}(z, w)=\left(|z|^{2}+|w|^{2}, 2 \operatorname{Re}(z \bar{w}), 2 \operatorname{Im}(z \bar{w}),|z|^{2}-|w|^{2}\right),
$$

and is called a universal rational parametrization of $S^{2}$ (see $[4,19]$ for details). Since $P_{S}: \mathbb{C}^{2} \rightarrow S^{2}$ is homogeneous $P_{S}(\lambda z, \lambda w)=|\lambda|^{2} P_{S}(z, w), \lambda \in \mathbb{C}, P_{S}$ defines also a map from a complex projective line $\mathbb{C} P^{1}$ to $S^{2}$, which is essentially the Riemann sphere construction.

The universal property of $P_{S}$ is formulated in the following theorem. Here we call a finite collection of polynomials $\left(f_{0}, f_{1}, \ldots\right)$ irreducible if $\operatorname{gcd}\left(f_{0}, f_{1}, \ldots\right)=1$.

TheOrem 5.1. Any irreducible solution $\mathbf{f}=\left(f_{0}, \ldots, f_{3}\right) \in \mathbb{R}\left[t_{1}, \ldots, t_{k}\right]^{4}$ of the unit sphere equation $f_{0}^{2}=f_{1}^{2}+f_{2}^{2}+f_{3}^{2}$ has the form $\mathbf{f}=P_{S}(\mathbf{F})$ with an irreducible $\mathbf{F}=(z, w) \in \mathbb{C}\left[t_{1}, \ldots, t_{k}\right]^{2}$, which is determined uniquely up to a complex constant multiplier $\lambda,|\lambda|=1$.

We call $\mathbf{F}=(z, w)$ a lifting of $\mathbf{f}$ and denote it by $\widetilde{\mathbf{f}}=\mathbf{F}$. The lifting can be calculated using a simple formula $[20]$

$$
\widetilde{\mathbf{f}}=\left(h\left(f_{0}+f_{3}\right) /\left(f_{1}-f_{2} \mathrm{i}\right), h\right), \quad h=\operatorname{gcd}\left(f_{1}-f_{2} \mathrm{i}, f_{0}-f_{3}\right) .
$$

The formula (5.3) enables the lifting of rational Bézier curves of degree $2 k$ and tensor product patches of degree $(2 k, 2 l)$ on $S^{2}$ to the corresponding polynomial curves of degree $k$ and surfaces of bi-degree $(k, l)$ in $\mathbb{C}^{2}$, respectively. This universal rational parametrization technique was used to find Bézier patches on $S^{2}$ of minimal degree with given boundary curves, see [19]. Theorem 5.1 can be applied for polynomials of arbitrary number of variables.

EXAMPLE 2. Consider a parametrization $\mathbf{f}$ which is the opposite on $S^{2}$ to $P_{S}(z, w)$ :

$$
\mathbf{f}=\left(-|z|^{2}-|w|^{2}, 2 \operatorname{Re}(z \bar{w}), 2 \operatorname{Im}(z \bar{w}),|z|^{2}-|w|^{2}\right)
$$

Let us calculate a lifting: $h=\operatorname{gcd}\left(2 \bar{z} w,-2|z|^{2}\right)=\bar{z}$, and $\widetilde{\mathbf{f}}=\left(\bar{z}\left(-2|w|^{2}\right) /(2 \bar{z} w), \bar{z}\right)=(-\bar{w}, \bar{z})$. Therefore, a point $P_{S}(-\bar{w}, \bar{z})$ is the opposite of $P_{S}(z, w)$. 
Projectively the Blaschke cylinder $\mathcal{B}$ is a cone over a sphere, since its equation is $y_{0}^{2}=$ $y_{1}^{2}+y_{2}^{2}+y_{3}^{3}$ ( $y_{4}$ is arbitrary) in $\mathcal{M} P$. In complex setting this equation can be transformed to the binomial one $\left(y_{0}-y_{3}\right)\left(y_{0}+y_{3}\right)=\left(y_{1}-\mathrm{i} y_{2}\right)\left(y_{1}+\mathrm{i} y_{2}\right)$. Therefore $\mathcal{B}$ is a real part of a toric variety (see e.g. [49]). According to a general theory [4], $\mathcal{B}$ has the universal rational parametrization in the slightly more complicated form:

$$
P_{B}(z, w, f, g)=\left(g\left(|z|^{2}+|w|^{2}\right), 2 g \operatorname{Re}(z \bar{w}), 2 g \operatorname{Im}(z \bar{w}), g\left(|z|^{2}-|w|^{2}\right), f\right) .
$$

The map $P_{B}: \mathbb{C}^{2} \times \mathbb{R}^{2} \rightarrow \mathbb{R}^{5}$ is homogeneous, $P_{B}((\lambda, \rho) *(z, w, f, g))=|\lambda|^{2} \rho P_{B}(z, w, f, g)$, with respect to the following multiplication:

$$
(\lambda, \rho) *(z, w, f, g)=\left(\lambda z, \lambda w,|\lambda|^{2} \rho f, \rho g\right), \quad \lambda \in \mathbb{C}, \rho \in \mathbb{R} .
$$

THEOREM 5.2. Any irreducible solution $\mathbf{h}=\left(h_{0}, \ldots, h_{4}\right) \in \mathbb{R}\left[t_{1}, \ldots, t_{k}\right]^{4}$ of the Blaschke cylinder equation $h_{0}^{2}=h_{1}^{2}+h_{2}^{2}+h_{3}^{2}$ has the form $\mathbf{h}=P_{B}(\mathbf{H})$, where $\mathbf{H}=(z, w, f, g) \in$ $\mathbb{C}\left[t_{1}, \ldots, t_{k}\right]^{2} \times \mathbb{R}\left[t_{1}, \ldots, t_{k}\right]^{2}$ and the pairs $(z, w)$ and $(f, g)$ are irreducible. $\mathbf{H}$ is determined uniquely up to multiplication by $(\lambda, \rho)$ defined in (5.5), with $|\lambda|^{2} \rho=1$.

EXAMPLE 3. Consider the particular parametrization of the Blaschke cylinder $\iota: I^{3} \rightarrow \mathcal{B}:$

$$
\iota: I^{3}=\mathbb{R}^{3} \cup \mathbb{R} \rightarrow B, \quad(u, v, f) \mapsto P_{B}(u+v \mathrm{i}, 1, f, 1), f \mapsto P_{B}(1,0, f, 1),
$$

which is called the isotropic model of Laguerre geometry. The composition with the Blaschke map $\Lambda=\iota^{-1} \circ \beta$ describes the change from the Euclidean model to the isotropic model. It will be useful for modeling applications in Section 7.1.

6. Special cases of PN surfaces. Important examples of PN surfaces are generated as envelopes of rational one parameter families of simplest primitive shapes: planes, spheres or circular cones (cylinders).

Envelopes of planes are exactly developable surfaces. Developable PN surfaces were already characterized by Theorem 2.2 as rational curves in the Blaschke cylinder (see Section 2.3). The other classes of PN surfaces are now discussed in the subsequent sections.

6.1. Canal surfaces. A canal surface is the envelope of one-parameter family of spheres in $\mathbb{R}^{3}$ defined by a spine curve $\mathbf{m}(t)$ and a radius function $r(t)$. In 1995 Lü [27] proved the surprising result: A canal surface defined by a rational spine curve and a rational radius function is rational. See also [28] for the details of the proof.

Later this result was proved by different methods: geometric approach [34], Clifford algebra formalism [2, 3] and a universal rational parametrization of a sphere [20]. Here we describe the approach in $[34,20]$ which gives bounds of rational parametrization degree.

As it was explained in Section 4.4, a canal surface is the cyclographic image $\gamma(\mathbf{s})$ of a curve $\mathbf{s}(t)=(\mathbf{m}, r)(t)$ in $\mathcal{M}$. It is real exactly if $\langle\dot{\mathbf{s}}(t), \dot{\mathbf{s}}(t)\rangle \geq 0$ (see (4.8)), and its isotropic hypersurface $\Gamma(\mathbf{s})$ consists of all isotropic lines that intersect the curve orthogonally. Therefore, we can look for a parametrization of $\Gamma(\mathbf{s})$ in the form $\mathbf{F}(s, t, \lambda)=\mathbf{s}(t)+\lambda \mathbf{n}^{+}(s, t)$, where $\left\langle\dot{\mathbf{s}}(t), \mathbf{n}^{+}(s, t)\right\rangle=0$. The latter condition means that isoparametric curves $\mathbf{a}_{t}(s)=\mathbf{n}(s, t)$ of a Gauss map $\mathbf{n}(s, t)$ define a family of circles as planar sections of the unit sphere $S^{2}$,

$$
\left\langle\dot{\mathbf{s}}(t), \mathbf{x}^{+}\right\rangle=0, \quad \mathbf{x}^{+}=(\mathbf{x}, 1), \mathbf{x} \in \mathbb{R}^{3} .
$$

If such a rational Gauss map $\mathbf{n}(s, t)$ exists then a rational parametrization $\mathbf{f}(s, t)$ of the canal surface $\gamma(\mathbf{s})=\Gamma(\mathbf{s}) \cap \mathbb{R}^{3}$ can be calculated by substitution $\mathbf{f}(s, t)=\mathbf{F}\left(s, t,-s_{4}(t)\right)$. Equivalently, $\mathbf{f}(s, t)$ can be expressed in terms of the spine curve $\mathbf{m}(t)$ and the radius function $r(t)$

$$
\mathbf{f}(s, t)=\mathbf{m}(t)-r(t) \mathbf{n}(s, t) .
$$

Consider the slightly more general case. Let $\Pi_{t}:\left\langle\mathbf{v}(t), \mathbf{x}^{+}\right\rangle=0$ be a family of planes with polynomial coefficients $\mathbf{v}(t)$, where $D(t)=\langle\mathbf{v}(t), \mathbf{v}(t)\rangle \geq 0, D(t) \not \equiv 0$. Then the polynomial $D(t)$ can be factorized

$$
D(t)=\prod_{i}\left(t-z_{i}\right)^{p_{i}}\left(t-\bar{z}_{i}\right)^{p_{i}} \rho(t)^{2}, \quad z_{i} \in \mathbb{C} \backslash \mathbb{R}, \quad \rho(t) \in \mathbb{R}[t] .
$$


THEOREM 6.1 ([20]). There exists a rational parametrization $\mathbf{n}(s, t)$ of the unit sphere $S^{2}$ of bidegree $(2, n)$, such that all isoparametric curves $\mathbf{a}_{t}(u)=\mathbf{n}(s, t)$ are plane sections $S^{2} \cap \Pi_{t}$. There is a constructive method for finding such parametrization $\mathbf{n}(s, t)$ with

$$
n=2 \max \left(\lceil m / 2\rceil, m-\sum_{i}\left\lceil p_{i} / 2\right\rceil\right), \quad m=\operatorname{deg} \mathbf{v}(t),
$$

where $p_{i}$ are multiplicities of complex roots of the polynomial $D(t)$ defined in (6.3).

The proof is based on the universal rational parametrization of the sphere (5.2), where $P_{S}$ is treated as a map $\mathbb{R} P^{3} \rightarrow S^{2}$. For any representation of $D(t)$ as a sum of squares there is a minimal solution $\mathbf{n}(s, t)=P_{S}(\mathbf{r}(s, t))$, which is an image of a certain ruled surface $\mathbf{r}(s, t)$ of implicit degree $m$ in $\mathbb{R} P^{3}$. Minimal directrices of $\mathbf{r}(s, t)$ with complimentary degrees $m_{0}+m_{1}=m$ can be found using $\mu$-basis methods [1]. The goal is to find the minimal $n=2 \max \left(m_{0}, m_{1}\right)$ in the list of all minimal solutions, which is achieved by a constructive procedure up till the value (6.4).

REMARK 6.1. The related problem of finding a decomposition of a real polynomial as a sum of two squares over $\mathbb{Q}$ was considered in [25]. It was proved that the problem is equivalent to partial factorization of of the polynomial, and a decomposition algorithm was presented in case the solution is defined over $\mathbb{Q}$.

Theorem 6.1 can be applied to a family of planes $(6.1)$ with $\mathbf{v}(t)=d(t) \dot{\mathbf{c}}(t)$, when $d(t)$ is a common denominator of all rational $\dot{c}_{i}(t), i=1, \ldots, 4$. If $\mathbf{c}(t)$ is a rational curve of degree $k$ then in general $\operatorname{deg} \mathbf{v}(t)=2 k-2$. Using the inequality $\operatorname{deg}_{t} \mathbf{f}(s, t) \leq \operatorname{deg} \mathbf{c}(t)+\operatorname{deg}_{t} \mathbf{n}(s, t)(\operatorname{see}(6.2))$ one can derive the following degree bounds.

Corollary 6.1 ([20]). A canal surface $\gamma(\mathbf{c})$ defined by a rational curve $\mathbf{c}(t)$ in $\mathbb{R}^{4}$ of degree $k$ admits a rational parametrization $\mathbf{f}(s, t)$ of bidegree $(2, n)$, where

(i) $n=3 k-2$ if $D(t)>0$ (all roots are complex),

(ii) $n=5 k-6$ if $D(t)$ has at least one complex root.

(iii) $n=5 k-4$ if $D(t)$ has all real roots.

The case (i) of this corollary gives in general the minimal possible degree, and the case (ii) was proved in [34]. The following example shows that and the case (iii) cannot be improved.

ExAmple 4. Consider a canal surface $\gamma(\mathbf{c})$ (see Fig. 2 middle) defined by the curve

$$
\mathbf{c}(t)=\left(0, \frac{1-t^{2}}{2\left(1+t^{2}\right)}, \frac{t}{1+t^{2}},-\frac{t}{1+t^{2}}\right) .
$$

Then $\mathbf{v}(t)=\left(1+t^{2}\right)^{2} \dot{\mathbf{c}}(t)=\left(0,-2,1-t^{2},-1+t^{2}\right)$ and $D(t)=4 t^{2}$ has no complex roots. Therefore there is only one factorization of $D(t)$ that defines a unique minimal parametrization $\mathbf{n}(t, u)=P_{S}((1-s) X+s Y)$, with $X=\left(\mathrm{i}\left(t^{2}-1\right), 2 t\right), Y=(1,0)$, of degree $(2,4)$. The canal surface $\gamma(\mathbf{c})$ is parametrized by $\mathbf{f}(s, t)$ (see $(6.2)$ ) of bidegree $(2,6)$, which is minimal possible.
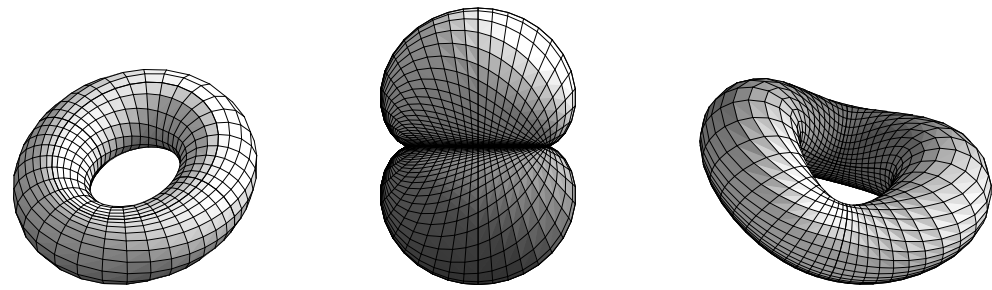

FIG. 2. Examples of canal surfaces of bidegree $(2,4),(2,6),(2,10)$.

6.2. Rational ruled surfaces in $\mathcal{M}$. Rational ruled surfaces in $\mathbb{R}^{3}$ are $P N$ surfaces if they are non-developable. This result was proved in $[35,31]$ and generalized to any rational ruled surface $\Psi$ in $\mathcal{M}$ in the sense that its cyclographic image $\gamma(\Psi)$ in $\mathbb{R}^{3}$ is rational.

The Blaschke model will be most convenient for understanding this result. Let a rational ruled surface $\Psi$ in $\mathcal{M}$ be defined by two directrices $\mathbf{c}(t)$ and $\mathbf{d}(t)$. The key idea is to construct a Gaussian map $\mathbf{n}(s, t)$ which for any fixed $t$ defines normals along a common touching curve 
between the envelope surface $\gamma(\Psi)$ and a cone of revolution defined by a line going through points $\mathbf{c}(t)$ and $\mathbf{d}(t)$. This is equivalent to the condition

$$
\left\langle\mathbf{n}^{+}(s, t), \mathbf{d}(t)-\mathbf{c}(t)\right\rangle=0
$$

which forces isoparametric curves of $\mathbf{n}(s, t)$ to be the prescribed family of circles. Therefore, such $\mathbf{n}(s, t)$ can be generated using Theorem 6.1. Then a support function $h(s, t)$ is computed from the equation

$$
h(s, t)+\left\langle\mathbf{n}^{+}(s, t), \mathbf{c}(t)\right\rangle=0 .
$$

Finally it remains to go back from the Blaschke image to $\gamma(\Psi)$, i.e. to compute the envelope of tangent planes $h(s, t)+\langle\mathbf{n}(s, t), \mathbf{x}\rangle=0$ in $\mathbb{R}^{3}$.

EXAMPLE 5 . Let $\Psi$ be a hyperbolic paraboloid $x_{3}=x_{1} x_{2}$ in $\mathbb{R}^{3}$ with two directrices $\mathbf{c}(t)=$ $(0, t, 0,0)$ and $\mathbf{d}(t)=(1, t, t, 0)$. The Gaussian map can be calculated as

$$
\begin{aligned}
\mathbf{n}(s, t) & =P_{S}(-s t+\mathrm{i}(1-s), 1-3 s-\mathrm{i} s t) \\
& =\left(-4 s t+8 s^{2} t,-2 s^{2} t^{2}+2-8 s+6 s^{2}, 4 s-8 s^{2}\right) /\left(1-4 s+5 s^{2}+s^{2} t^{2}\right) .
\end{aligned}
$$

One can check directly that the condition (6.5) is fulfilled. Then the Blaschke image is derived using (6.6), and finally a point representation of any offset of $\Psi$ is generated of bidegree $(4,5)$. Compare with a parametrization of bidegree $(6,6)$ that is generated by treating $\Psi$ as $L N$ surface (see Section 3).

6.3. Characterization of $\mathrm{PN}$ surfaces of low parametrization degree. For a PN surface $F$ parametrized by $\mathbf{f}(s, t)$ consider the parametrization of its isotropic hypersurface $\Gamma(F)$ in the form $\mathbf{F}(s, t, u)=\mathbf{f}(s, t)+u \mathbf{n}^{+}(s, t)$ (see Section 6.1), and a family $\Phi_{t}$ of its isoparametric ruled subsurfaces $\Phi_{t}(s, u)=\mathbf{F}(s, t, u)$. Define the $P N$ degree of the PN parametrization of $F$ with respect to $s$ as implicit degree of $\Phi_{t}$. Then any general Laguerre transform of $\mathbf{f}(s, t)$ (see Section 4.3) will have the same PN degree in $s$.

It was shown in previous Sections 6.1 and 6.2 that the simplest PN surfaces admit parametrizations $\mathbf{f}(s, t)$ with $\operatorname{deg}_{s}(\mathbf{f}) \leq 4$. Now we are going to show that they can be almost characterized by this degree.

TheOREM 6.2. If a $P N$ surface $F$ parametrized by $\mathbf{f}(s, t)$ has $P N$ degree $m \leq 4$ in $s$ then $F$ $i s$ :

(i) a developable PN surface if $m=1$;

(ii) a rational canal surface if $m=2$;

(iii) an envelope of a rational family of circular cones if $m=3$;

(iv) an envelope of a rational family of circular cones or Dupin cyclides if $m=4$.

Proof. If $m=1$ then the surface $\Phi_{t}$ is a plane and its projection to $\mathbb{R}^{3}$ is also a plane containing normals $\mathbf{n}(s, t)$ along the line $\Phi_{t}(s, 0)$. Hence $\mathbf{n}(s, t)$ is constant for any fixed $t$, and $F$ is developable.

In the case $m=2$ the surface $\Phi_{t}$ is a quadric that spans a time-like hyperplane in $\mathcal{M}$, that can be moved to a standard position $x_{3}=0$ using an appropriate Laguerre transformation. Then all normals along the conic $\Phi_{t}(s, 0)$ will be in the same plane. Thus these conics are circles and $\Phi_{t}$ are cones with vertices $\mathbf{c}(t)$ that trace a curve in $\mathcal{M}$. Then $F$ is a canal surface $\gamma(\mathbf{c})$.

In the case $m=3$ the surface $\Phi_{t}$ is a ruled cubic, which cannot be a cone (since otherwise its vertex belongs to $\Omega$ and $F$ is developable). Then there is exactly one linear directrix $\mathbf{l}_{t}$ in $\Phi_{t}$ for every parameter $t$, i.e. they define a rational family of circular cones $\gamma\left(\mathbf{l}_{t}\right)$ with the envelope $F$.

In the last case $m=4$ the surface $\Phi_{t}$ is a ruled quartic. By the same arguments as above this cannot be a cone. Then $\Phi_{t}$ has a family of conics $\mathbf{c}_{v}$ as directrices. Since all canal surfaces $\gamma\left(\mathbf{c}_{v}\right)$ are touching along a common quartic curve $\Phi_{t}(s, 0)$, conics $\mathbf{c}_{v}$ have only space-like tangents. Such canal surfaces have been studied in [24], where it has been proved that there exists a circular cone in the family $\gamma\left(\mathbf{c}_{v}\right)$, except in the Dupin cyclide case (see [24], Corollary 2). Therefore $F$ is an envelope of these circular cones.

Examples with $m=3,4$ are provided by branching blend surfaces of bidegree $(3,6)$ and $(4,8)$ in Section 7.4 (see also [21]). The latter case corresponds to a family of Dupin cyclides. 
6.4. Offsets of regular quadric surfaces in $\mathbb{R}^{3}$. Regular quadrics are one of the simplest surfaces in $\mathbb{R}^{3}$. Nevertheless it is not obvious that their offsets admit rational parametrizations. Investigating conics in the plane it is quite clear that the offset curves of ellipses and hyperbolas are non-rational whereas the offsets of circles and parabolas are rational curves. Lü [30] has been the first who proved that the offsets of all regular quadrics admit rational parametrizations. For the paraboloids this is not difficult, for ellipsoids and hyperboloids this is quite involved.

The existence of rational parametrizations of the offsets of regular quadrics can be shown as follows. Let $\Phi$ be a two-dimensional quadric in $\mathcal{M}$, then $\Phi$ is contained in a hyperplane of $\mathcal{M}$. We are studying the isotropic hypersurface $\Gamma(\Phi)$ corresponding to $\Phi$. The intersection of $\Gamma(\Phi)$ with $x_{4}=0$ is the cyclographic image $\gamma(\Phi)$, the envelope of the two-parameter family of oriented spheres corresponding to $\Phi$. If $\Phi$ is contained in a hyperplane $x_{4}=d$, the envelope $\gamma(\Phi)$ is the offset surface of $\Phi$.

The isotropic hypersurface $\Gamma(\Phi)$ is the envelope of common tangent hyperplanes of the pencil of quadrics $\lambda \Phi+\mu \Omega$ in $\mathcal{M} P$. The quadrics $\Phi$ and $\Omega$ are considered as sets of tangent hyperplanes, which implies that they are singular hypersurfaces in this pencil.

The intersection surface of two hyperquadrics in $\mathcal{M} P$ is a rational quartic del Pezzo surface. This del Pezzo surface is dual to the isotropic hypersurface $\Gamma(\Phi)$. This implies that the two parameter family of tangent hyperplanes of $\Gamma(\Phi)$ can be rationally parametrized. Intersecting $\Gamma(\Phi)$ with $x_{4}=0$ gives a parametrization of $\gamma(\Phi)$ as set of tangent planes. This construction proves the following result.

Proposition 6.1. The cyclographic images $\gamma(\Phi)$ of two dimensional quadrics $\Phi \subset \mathcal{M}$ are surfaces admitting rational parametrizations.

We demonstrate also an alternative way to construct rational PN parametrizations of the offset surfaces of quadrics in $\mathbb{R}^{3}$. Let $\Phi$ be a quadric surface in $\mathcal{M}$, contained in a space-like hyperplane, for instance $x_{4}=0$. We show that the pencil of quadrics $\lambda \Phi+\mu \Omega$ in $\mathcal{M} P$ contains a ruled quadric surface $\Psi$ and $\Gamma(\Phi)=\Gamma(\Psi)$ holds. Then the cyclographic images $\gamma(\Phi)$ and $\gamma(\Psi)$ agree and rational parametrizations can be constructed as described in Section 6.2.

Theorem 6.3. All regular quadrics are PN surfaces.

Let $\Phi$ be a regular quadric possessing real points. Quadrics of revolution are canal surfaces and thus the rationality of their offsets follows from Section 6.1. If $\Phi$ itself is a ruled quadric surface in $\mathcal{M}$, we may directly apply the method outlined in Section 6.2.

Otherwise let $\Phi$ be contained in the hyperplane $x_{4}=0$. The pencil of dual hyperquadrics $\lambda \Phi+\mu \Omega$ in $\mathcal{M}$ defines the isotropic hypersurface $\Gamma(\Phi)$. All singular quadrics in this pencil possess the same isotropic hypersurface $\Gamma(\Phi)$. The offset surfaces of $\Phi$ at distance $d$ are obtained as hyperplane sections $\Gamma(\Phi) \cap\left\{x_{4}=d\right\}$. We will find a real ruled quadric $\Psi$ in all of the three cases which have to be discussed.

- Let $\Phi$ be an ellipsoid in $x_{4}=0$. Then $\Phi$ (with $a>b>c$ ) and $\Psi$ are given by the equations

$$
\Phi: \frac{x_{1}^{2}}{a^{2}}+\frac{x_{2}^{2}}{b^{2}}+\frac{x_{3}^{2}}{c^{2}}=1, x_{4}=0, \quad \Psi: \frac{x_{1}^{2}}{a^{2}-b^{2}}-\frac{x_{3}^{2}}{b^{2}-c^{2}}+\frac{x_{4}^{2}}{b^{2}}=1, x_{2}=0 .
$$

- Let $\Phi$ be a two sheet hyperboloid in $x_{4}=0$. Then $\Phi$ (with $b>c$ ) and $\Psi$ are given by the equations

$$
\Phi: \frac{x_{1}^{2}}{a^{2}}-\frac{x_{2}^{2}}{b^{2}}-\frac{x_{3}^{2}}{c^{2}}=1, x_{4}=0, \quad \Psi: \frac{x_{1}^{2}}{a^{2}+b^{2}}+\frac{x_{3}^{2}}{b^{2}-c^{2}}-\frac{x_{4}^{2}}{b^{2}}=1, x_{2}=0 .
$$

- Let $\Phi$ be an elliptic paraboloid in $x_{4}=0$. Then $\Phi$ (with $b>c$ ) and the hyperbolic paraboloid $\Psi$ are given by the equations

$$
\Phi: \frac{x_{1}^{2}}{a^{2}}+\frac{x_{2}^{2}}{b^{2}}-2 x_{3}=0, x_{4}=0, \quad \Psi:-\frac{1}{a^{2}-b^{2}} x_{2}^{2}+\frac{1}{a^{2}} x_{4}^{2}=2 x_{3}-a, x_{1}=0 .
$$



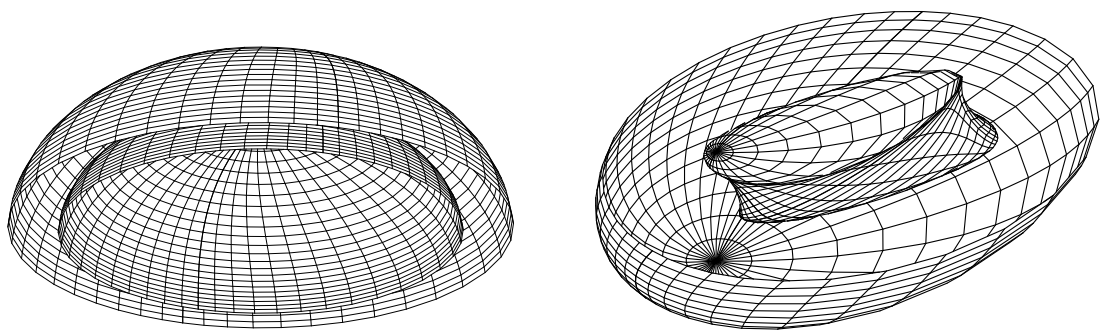

FiG. 3. Left: Ellipsoid of revolution and outside offset. Right: General ellipsoid and inside offset.

6.5. Quadratic triangular Bézier surfaces in $\mathcal{M}$. In Section 6.2 it has been proved that the cyclographic images $\gamma(F)$ of rational ruled surfaces $F$ in $\mathcal{M}$ are PN surfaces. Besides this result not much has been known about rationality of cyclographic images. Recently it has been proved in [36] that any quadratic triangular Bézier surface in $\mathcal{M}$ possesses a rational envelope surface of the corresponding family of spheres. This result can directly be proved starting with an appropriate parametrization $\mathbf{w}(s, t)$ of the absolute quadric $\Omega$ and solving the equations (4.7).

Let

$$
\mathbf{f}(u, v)=\frac{1}{2} \mathbf{a}_{1} u^{2}+\mathbf{a}_{2} u v+\frac{1}{2} \mathbf{a}_{3} v^{2}+\mathbf{a}_{4} u+\mathbf{a}_{5} v+\mathbf{a}_{6}, \text { with } \mathbf{a}_{i} \in \mathbb{R}^{4}
$$

be a parametrization of a quadratic triangular Bézier surface $F$ spanning $\mathbb{R}^{4}$. For convenience we use the monomial basis instead of the Bernstein basis for the representation of $F$.

In order to solve (4.7), we may start with a rational parametrization $\mathbf{w}(s, t)$ of $\Omega$, which obviously satisfies $\langle\mathbf{w}, \mathbf{w}\rangle=0$. A possible choice is $\mathbf{w}(s, t)=\left(2 s, 2 t, 1-s^{2}-t^{2}, 1+s^{2}+t^{2}\right)$. The conditions $\left\langle\mathbf{w}, \mathbf{f}_{u}\right\rangle=0$ and $\left\langle\mathbf{w}, \mathbf{f}_{v}\right\rangle=0$ are linear in $u$ and $v$. Thus, a solution of the system of linear equations

$$
\left(\begin{array}{cc}
\left\langle\mathbf{w}, \mathbf{a}_{1}\right\rangle & \left\langle\mathbf{w}, \mathbf{a}_{2}\right\rangle \\
\left\langle\mathbf{w}, \mathbf{a}_{2}\right\rangle & \left\langle\mathbf{w}, \mathbf{a}_{3}\right\rangle
\end{array}\right)\left(\begin{array}{c}
u \\
v
\end{array}\right)=\left(\begin{array}{l}
-\left\langle\mathbf{w}, \mathbf{a}_{4}\right\rangle \\
-\left\langle\mathbf{w}, \mathbf{a}_{5}\right\rangle
\end{array}\right)
$$

is a rational reparametrization $u=a(s, t), v=b(s, t)$ for the quadratic triangular Bézier surface $F$. It can be proved that the determinant of the coefficient matrix of (6.9) does not vanish identically expect for quadratically parameterized planes $F$. The isotropic lines $i(s, t): \mathbf{f}(s, t)+\lambda \mathbf{w}(s, t)$ are solutions of (4.7) and form a rational parametrization of the isotropic hypersurface $\Gamma(F)$ through $F$. The intersection $\Gamma(F) \cap \mathbb{R}^{3}$ is the envelope $\gamma(F)$ of the two-parameter family of spheres corresponding to $F$.

7. Modeling applications. Our first non-trivial modeling applications of surfaces with rational offsets are related to Dupin cyclides (see Fig. 4, left). These are special canal surfaces which are cyclographic images of Minkowski circles, i.e. conics in $\mathcal{M}$ that intersect $\Omega$ (see details in $[5,23])$. Dupin cyclides were proposed to be used as blending surfaces between natural quadrics by Pratt [46, 47] (see Fig. 4). For example, any two circular cones with a common inscribed sphere can be blended by a part of a Dupin cyclide bounded by two circles (Fig. 4, middle). In terms of the cyclograpic model this is a simple rounding of two intersecting space-like lines by an arc of a Minkowski circle. Similar blending is available between a circular cylinder (or cone) and a plane (Fig. 4, right).

More sophisticated modeling schemes with patches of Dupin cyclides bounded by circles were considered in [50]. A very special of blending between two circular cylinders using parabolic Dupin cyclides was proposed in [51].

7.1. Modeling with parabolic cyclides. The technique described in Example 1 can be used to develop a surface modeling scheme based on parabolic Dupin cyclides in [33] . Let scattered data elements $\left(\mathbf{a}_{i}, A_{i}\right)$ be given in $\mathbb{R}^{3}$, where $\mathbf{a}_{i}$ are vertices incident with the oriented planes $A_{i}$. The goal is to construct a $C^{1} \mathrm{PN}$ surface, which interpolates the given data and which is composed 

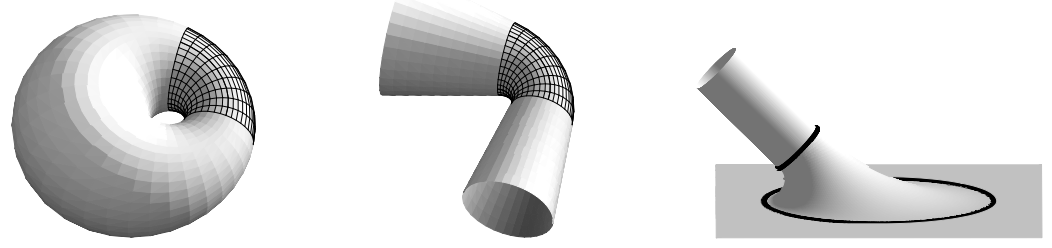

FIG. 4. Using patches of cyclides for blending.

of triangular patches of parabolic Dupin cyclides. The concept is the following. The data $\left(\mathbf{a}_{i}, A_{i},\right)$ are mapped by $\Lambda$ to the isotropic model $I^{3}$ (see also Example 3). The images are scattered data elements, say $\left(\mathbf{b}_{i}, B_{i}\right)$, with $\Lambda\left(A_{i}\right)=B_{i}$. The data $\left(\mathbf{b}_{i}, B_{i}\right)$ will be interpolated by a $C^{1}$ function $\Psi$, which is piecewise quadratic, using the method of Powell-Sabin [45]. Returning to the standard model we obtain a $C^{1}$ interpolating surface $\Lambda^{-1}(\Psi)$ composed of parabolic Dupin cyclides. We note that in general the triangular cyclide pieces are tangent to each other along cubics and not along circles. This already indicates that this method is rather different from other surface modeling schemes, using (parabolic) Dupin cyclides, as [46, 50] and others. However, smooth surfaces with vanishing Gaussian curvature along curves other than straight lines can never be modeled with parabolic Dupin cyclides.

7.2. Approximations with developable PN surfaces. Very few applications of developable PN surfaces are known. In [26] developable surfaces are modeled with pieces of circular cones. A more general method for the recognition and reconstruction of developable surfaces was proposed in [32]. The approximation problem with given data points as measurements from a developable surface and estimated tangent planes is translated to a curve fitting problem which is solved on the Blaschke cylinder. Then the constructed curve is interpreted as one-parameter family of tangent planes and their envelope is calculated.

7.3. Blending natural quadrics with canal surfaces. Canal surfaces defined by general conics in $\mathcal{M}$ can be used for blending cones/cylinders in more general positions (Fig. 5) along quartic boundary curves as was shown in [24].
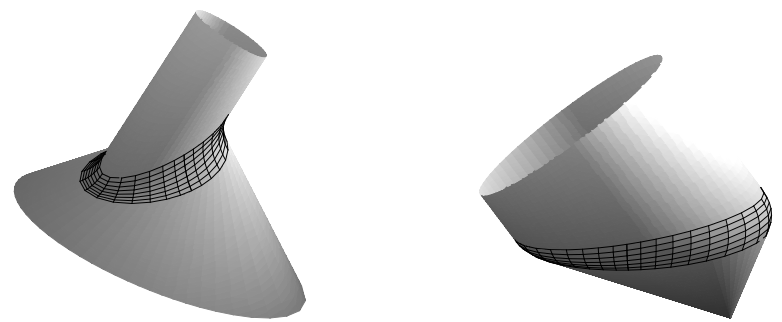

FIG. 5. Blendings of cylinders/cones of bidegree $(2,4)$ along quartic curves.

Results on rational parametrizations [34, 20] have been used in [15] to develop a theory on rational variable radius rolling ball blends between natural quadrics in arbitrary positions. Here we will consider just one illustrative example.

ExAmple 6 . Let $Q_{a}$ and $Q_{b}$ be two cylinders in $\mathbb{R}^{3}$ defined by equations $x_{1}^{2}+x_{2}^{2}=r_{a}^{2}$ and $x_{2}^{2}+x_{3}^{2}=r_{b}^{2}$, where $0<r_{a}<r_{b}$ (Fig. 6, right). Consider lines $L_{a}$ and $L_{b}$ in $\mathcal{M}$ such that their cyclographic images are given cylinders $Q_{a}$ and $Q_{b}$. Then an intersection of their isotropic hypersurfaces is a quartic surface $\Phi=\Gamma\left(L_{a}\right) \cap \Gamma\left(L_{b}\right)$ in $\mathcal{M}$ which projects exactly to the bisector of the cylinders in $\mathbb{R}^{3}$ (Fig. 6, left).

Any curve on $\Phi$ defines a canal surface touching both cylinders, i.e. a rolling ball blend. Unfortunately a fixed radius case corresponds to a non-rational curve on $\Phi$. Nevertheless, a certain rational quartic curve $\mathbf{s} \subset \Phi$ can be found [22]. This construction generates a canal surface of bidegree $(2,10)$ which is minimal possible according to Corollary 6.1 . It is impossible to construct 

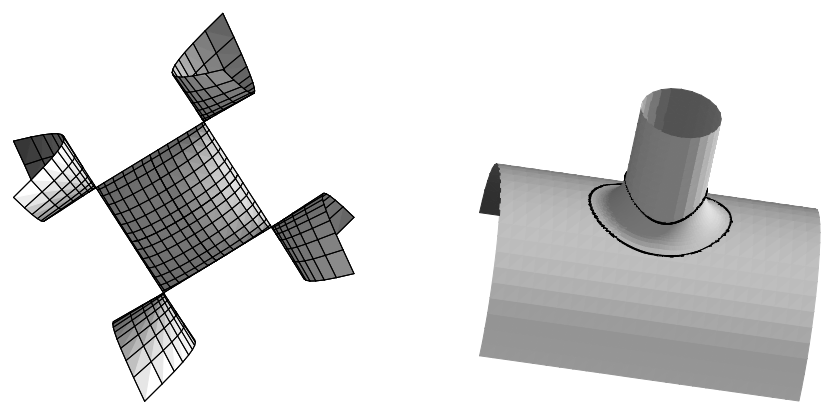

FIG. 6. Bisector of two cylinders and their blending of bidegree $(2,10)$.

such a blending with a boundary circle on the cylinder $Q_{a}$, since the corresponding curve on $\Phi$ and the associated canal surface are non-rational.

7.4. Branching blend of natural quadrics using non-canal PN surfaces. The blending in Example 6 can be improved by using $\mathrm{PN}$ surfaces that are more general than canal surfaces. Here we sketch the construction proposed in [21].

The goal is to generate a branching blend of cylinders $Q_{a}$ and $Q_{b}$ defined in Example 6, which is a $\mathrm{PN}$ surface bounded by a circle $C_{a}, x_{3}=h$, on the vertical cylinder $Q_{a}$ and by a rational curve $C_{b}$ on the upper side of the horizontal cylinder $Q_{b}$ (see Fig. 7, left).
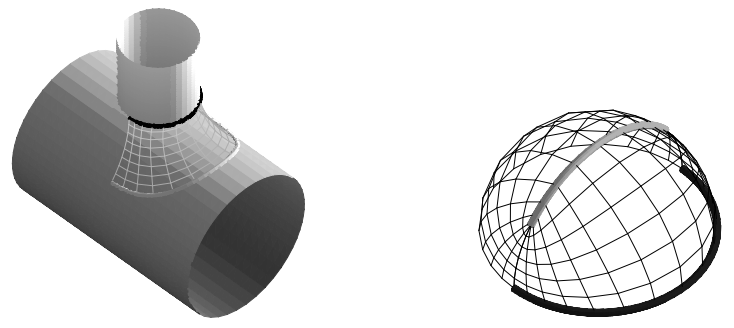

FIG. 7. Boundary curves $C_{a}, C_{b}$ and their images $\mathbf{n}_{a}, \mathbf{n}_{b}$ on the Gaussian sphere.

The general scheme of the proposed method consists of three steps.

Step 1: Gaussian map. Normals along $C_{a}$ and $C_{b}$ define two curves on the unit sphere: a circle $\mathbf{n}_{a}$ on the equator and a circular arc $\mathbf{n}_{b}$ on the plane section $x_{1}=0$, see Fig. 7 (right). In order to build a symmetric Gaussian map it remains to find a Bézier representation of the spherical quarter. Methods of [19] can be directly applied: a linear combination of the liftings $\widetilde{\mathbf{n}}_{a}$ and $\widetilde{\mathbf{n}}_{b}$ to $\mathbb{C}^{2}$ (see Section 5 )

$$
(z, w)=(1-s)(1-\mathrm{i} t) \widetilde{\mathbf{n}}_{a}+s \widetilde{\mathbf{n}}_{b}, \quad \widetilde{\mathbf{n}}_{a}=(1-\mathrm{i} t, t-\mathrm{i}), \widetilde{\mathbf{n}}_{b}=\left(1+t^{2}, 2 k t\right) .
$$

with the resulting unique parametrization in a homogeneous form $\mathbf{n}(s, t)=P_{S}(z, w)$ of degree $(2,4)$, where homogeneous coordinates are used $\mathbf{n}=\left(n_{0}, \ldots, n_{3}\right)$. Note, that the parameter $k$ controls the endpoints of the arc $\mathbf{n}_{b}$.

Step 2: Support function. Points $\mathbf{s}_{a}=\left(0,0, h, r_{a}\right)$ and $\mathbf{s}_{b}=\left(0,0,0, r_{b}\right)$ in Minkowski space $\mathcal{M}$ represent two spheres: touching the cylinder $Q_{a}$ along $C_{a}$, and the cylinder $Q_{b}$ along a circle with the normal $\mathbf{n}_{b}$. Their Blaschke images are constructed with the same fixed Gaussian map $\mathbf{n}(s, t)$ and represented in the universal rational parametrization form (5.4) with $g_{a}=g_{b}=1$ and certain polynomials $f_{a}$ and $f_{b}$ of bidegree $(2,4)$. The formula $f(s, t)=f_{a}(s, t)+s^{2}\left(f_{b}(1, t)-f_{a}(1, t)\right)$ defines a polynomial that is in $C^{1}$-contact with $f_{a}$ along $s=0$ and coincides with $f_{b}$ on $s=1$. Then the parametrization $(\mathbf{n}(s, t), f(s, t))=P_{B}(z, w, f, 1)$ is the dual of the blending solution.

Step 3: Back to the point representation. From the dual representation $e_{0}(s, t)+\langle\mathbf{e}(s, t), \mathbf{x}\rangle=$ $0, e_{0}=f, \mathbf{e}=\left(n_{1}, n_{2}, n_{3}\right)$, in Euclidean space we obtain the point representation by calculating the envelope (cf. (2.8)). If the dual data is of bidegree $\left(d_{t}, d_{u}\right)$ then the bidegree of the solution 
$\left(x_{1}, x_{2}, x_{3}\right)$ is $\left(3 d_{s}-2,3 d_{t}-2\right)$ in general. Since $\left(d_{s}, d_{t}\right)=(2,4)$ we can expect a solution of bidegree $(4,10)$. Fortunately there exists a unique value of $k$ in the expression of $\mathbf{n}_{b}$ (7.1) that enables us to drop bidegree down to $(3,6)$.

It was proved in [21] that this is the minimal possible Laguerre invariant bidegree. The construction can be generalized to a few other positions of the given cylinders and then extended to any possible position by applying appropriate Laguerre transformations. Moreover by applying inversions similar PN branching blends can be generated between Dupin cyclides and cylinders or cones of bidegree $(4,8)$. These possibilities are illustrated in Fig. 8.
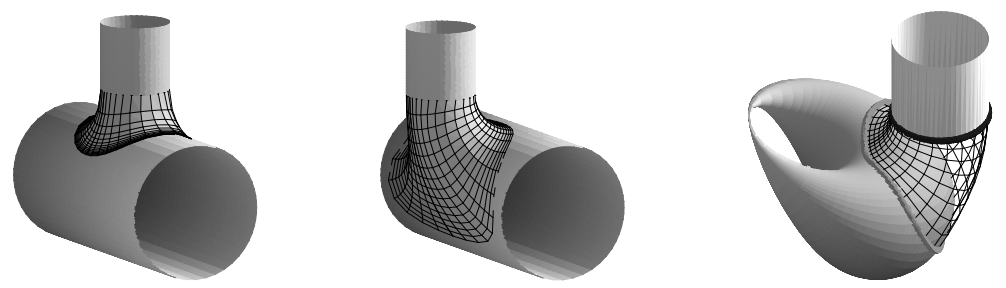

FIG. 8. Various branching blends between cylinders and Dupin cyclides.

8. Conclusions and open problems. We have given an overview of Pythagorean normal surfaces including their short history, an introduction to the language of Laguerre geometry, review of the most important classes of PN surfaces and their applications in geometric modeling. It has been shown that the dual approach in combination with universal rational parametrization ideas seems to be most promising not only for theoretical investigations but also for solving very practical modeling problems. There are still many questions that need to be discussed. Here are a few open problems for future research:

- Results on minimal parametrization degree of canal surfaces in Section 6.1 raises similar questions for other classes of PN surfaces. It is most important to understand possible reductions of degree when going from the dual representation to the point representation. Similar problems for PH curves were considered in [11].

- A free-form modeling scheme with PN surfaces without restrictions on the Gaussian curvature is a challenge. Perhaps the modeling scheme with parabolic cyclide patches (see Section 7.1) can be generalized using the universal rational parametrization of the Blaschke cylinder.

- Pieces of isotropic hypersurfaces of PN surfaces (e.g. between a surface and its offset) in Minkowski space projected down to Euclidean space $\mathbb{R}^{3}$ give nice examples of 3D rational parametrizations of solids. Sufficient non-degeneracy conditions of such parametrizations will be useful in modeling.

We hope that this survey will help interested researches and people from industry to get an adequate impression about achieved results and the state of art in investigations of PN surfaces and their modeling applications.

\section{REFERENCES}

[1] Chen, F., (2003). Reparametrization of a rational ruled surface using the $\mu$-basis, Computer Aided Geometric Design Vol. 20, pp. 11-17.

[2] Choi, H.I., LeE, D.S., Moon, H.P. (2002), Clifford algebra, spin representation, and rational parametrizations of curves and surfaces, Advances in Computational Mathematics 17, 2002, pp. 5-48.

[3] Cho, H.C., Choi, H.I., Kwon, H.-S., Lee, D.S., Wee, N.-S., Clifford algebra, Lorentzian geometry, and rational parametrizations of canal surfaces, Computer Aided Geometric Design 21, 2004, pp. 327-339.

[4] Cox, D., Krasauskas, R., Mustaţă, M., Universal rational parametrizations and toric varieties, Topics in Algebraic Geometry and Geometric Modeling, Contemporary Mathematics 334, 2003, pp. 241-265.

[5] Degen, W., Cyclides, in: G. Farin, J. Hoschek, M.-S. Kim (eds.) Handbook of Computer Aided Geometric Design, Elsevier Science, 2002, pp. 575-602.

[6] Dietz, R., Hoschek, J., And Jüttler, B., An algebraic approach to curves and surfaces on the sphere and other quadrics, Computer Aided Geometric Design 10, 1993, pp. 211-229. 
[7] Farouki, R.T., Pythagorean-hodograph curves in practical use, in: Geometry Processing for Design and Manufacturing, (Barnhill, R.E., ed.), SIAM, Philadelphia, 1992, pp. 3-33.

[8] Farouki, R.T., 2002. Pythagorean Hodograph curves, in: Handbook of Computer Aided Geometric Design, (Farin, G., Hoschek, J., Kim, M.-S. eds.), Elsevier, 2002.

[9] Farouki, R.T., Pythagorean-hodograph curves: algebra and geometry inseparable, Geometry and Computing Vol. 1, Springer, 2008.

[10] Farouki, R.T. and NefF, C.A., Algebraic properties of plane offset curves, Computer Aided Geometric Design 7, 1990, 101-127.

[11] Farouki, R.T. and Pottmann, H., Polynomial and rational Pythagorean-hodograph curves reconciled, in: G. Mullineux (ed.), The Mathematics of Surfaces VI, Oxford Univ. Press, 1996, pp. 355-378.

[12] Hoschek, J., Lasser, D., Fundamentals of Computer Aided Geometric Design, A. K. Peters, Wellesley, MA, 1993.

[13] JüttleR, B., 1998. Triangular Bézier surface patches with a linear normal vector field, in: The Mathematics of Surfaces VIII, Information Geometers, 431-446.

[14] Jüttler, B., Sampoli, M.L., Hermite interpolation by piecewise polynomial surfaces with rational offsets, Computer Aided Geometric Design 17, 2000, pp. 361-385.

[15] KaZAKevičIUTté, M., Blending of natural quadrics with rational canal surfaces, PhD Thesis, Vilnius University, 2005.

[16] M. KaZAKevičIŪtė, Classification of pairs of natural quadrics from the point of view of Laguerre geometry, Lithuanian Mathematical Journal 45, 2005, pp. 63-84.

[17] Kosinka, J. And Jüttler,B., MOS surfaces: Medial Surface Transforms with Rational Domain Boundaries, in: The Mathematics of Surfaces XII, R. Martin, M. Sabin, J. Winkler (eds.), Lecture Notes in Computer Science, Springer, to appear.

[18] Krasauskas, R., Universal parametrizations of some rational surfaces, in: A. Le Méhauté, C. Rabut and L.L. Schumaker (eds.): Curves and Surfaces with Applications in CAGD, Vanderbilt Univ. Press, Nashville, 1997, pp. 231-238.

[19] Krasauskas, R., Bézier patches on almost toric surfaces, in: Elkadi, M., Mourrain, B. and Piene, R. (eds.), Algebraic Geometry and Geometric Modeling, Mathematics and Visualization Series, Springer, 2006, pp. $135-150$

[20] Krasauskas, R., Minimal rational parametrizations of canal surfaces, Computing 79, 2007, pp. 281-290.

[21] Krasauskas, R., Branching blend of natural quadrics based on surfaces with rational offsets, Computer Aided Geometric Design 25, (2008), pp. 332-341.

[22] Krasauskas, R., KaZakeviČıūtè, M., Universal rational parametrizations and spline curves on toric surfaces, in: Computational Methods for Algebraic Spline Surfaces, ESF Exploratory Workshop, Springer, 2005, pp. 213-231.

[23] Krasauskas, R. And Mäurer, C., Studying cyclides using Laguerre geometry, Computer Aided Geometric Design 17, 2000, pp. 101-126.

[24] Krasauskas, R., Zube, S., Canal surfaces defined by quadratic families of spheres, Proceedings of the COMPASS II Workshop, 2007, pp. 138-150.

[25] Landsmann, G., Schicho, J. And Winkler F., The Parametrization of Canal Surfaces and the Decomposition of Polynomials into a Sum of Two Squares, Journal of Symbolic Computation 32, 2001, pp. 119-132.

[26] S. Leopoldseder, H. Pottmann, 1998. Approximation of developable surfaces with cone spline surfaces, Computer-Aided Design 30, 571582 .

[27] Lü, W., Rational canal surfaces, Technical Report No. 13, Institut für Geometrie, TU Wien, 1995.

[28] Lü, W. And Pottmann, H., Pipe surfaces with rational spine curve are rational, Computer Aided Geometric Design 13, 1996, pp. 327-339.

[29] Lü, W., 1994. Rationality of the offsets to algebraic curves and surfaces, Applied Mathematics 9 (Ser. B), 265-278.

[30] Lü, W., 1996. Rational parametrization of quadrics and their offsets, Computing 57, 1996, 135-147.

[31] Peternell, M., Rational parametrizations for envelopes of quadric families, Ph.D. Thesis, Institute of Geometry, University of Technology, Vienna, 1997.

[32] Peternell, M., Developable surface fitting to point clouds, Computer Aided Geometric Design 21, 2004, 785-803.

[33] Peternell, M. and Pottmann, H., Designing rational surfaces with rational offsets, Fontanella, F., Jetter, K., and Laurent, P.J. (eds.), 1996, pp. 275-286.

[34] Peternell, M. and Pottmann, H., Computing rational parametrizations of canal surfaces, J. Symbolic Computation 23, 1997, pp. 255-266.

[35] Peternell, M. and Pottmann, H., 1998. A Laguerre geometric approach to rational offsets, Computer Aided Geometric Design 15, 223-249.

[36] Peternell, M., Odehnal, B. and Sampoli, M.L.. On quadratic two-parameter families of spheres and their envelope, Computer Aided Geometric Design, to appear.

[37] Pottmann, H., 1994. Applications of the dual Bézier representation of rational curves and surfaces, in: Laurent, P.J., LeMéhauté, A. and Schumaker, L.L., eds., Curves and Surfaces in Geometric Design, A K Peters, Wellesley, MA, 377-384.

[38] Pottmann, H., 1995. Rational curves and surfaces with rational offsets, Computer Aided Geometric Design 12, $175-192$

[39] Pottmann, H., (1995). Curve design with rational Pythagorean-hodograph curves, Advances in Comp. Math. 
$3,147-170$.

[40] Pottmann, H., Studying NURBS curves and surfaces with classical geometry, in: Mathematical Methods for Curves and Surfaces, eds: M. Dæhlen and T. Lyche and L. L. Schumaker, Vanderbilt University Press, 1995, pp. 413-438.

[41] Pottmann, H. and Farin, G., 1995. Developable rational Bézier and B-spline surfaces, Computer Aided Geometric Design 12, 513-531.

[42] Pottmann, H., Lü, W. And Ravani, B., 1996. Rational ruled surfaces and their offsets, Graphical Models and Image Processing 58, 544-552.

[43] Pottmann, H. and Peternell, M., 1998. Applications of Laguerre Geometry in CAGD, Computer Aided Geometric Design 15, 165-186.

[44] Pottmann, H. And Wallner, J., Computational Line Geometry, Springer-Verlag, 2001.

[45] Powell, M.J.D. and Sabin, M., Piecewise quadratic approximation on triangles, ACM Transactions on Mathematical Software 3, 1977, pp. 317-325.

[46] Pratt, M. J., Cyclides in computer aided geometric design, Computer Aided Geometric Design 7, pp. 221242.

[47] Pratt, M. J., Cyclides in computer aided geometric design II, Computer Aided Geometric Design 12, 1995, pp. 131-152.

[48] Sampoli, M.L., Peternell, M. And Jüttler, B., Exact parametrization of convolution surfaces and rational surfaces with linear normals, Computer Aided Geometric Design 23, 2006, pp. 179-192.

[49] Sottile, F., Toric ideals, real toric varieties, and the moment map, Topics in Algebraic Geometry and Geometric Modeling, Contemporary Mathematics 334, 2003, pp. 225-240.

[50] Srivinas, Y.L. And Dutta, D., An intuitive procedure for constructing geometrically complex objects using cyclides, Computer-Aided Design 26, 1994, pp. 327-335.

[51] Ueda, K., Blending between right circular cylinders with parabolic cyclides, Proceedings of the Geometric Modeling and Processing 2000, April 10 - 12, 2000, pp. 390-397. 